D.O.I.: $10.3895 / \mathrm{S} 1808-04482009000100005$

\title{
METODOLOGIAS PROSPECTIVAS E O DESENVOLVIMENTO DE UM MODELO AUTO-REGRESSIVO PARA PREVISÃO DE DEMANDA NUMA INDÚSTRIA DE PLÁSTICO
}

\section{METHODOLOGIES OF PREDICTION AND THE DEVELOPMENT OF A AUTO-REGRESSIVE MODEL FOR FORECAST OF DEMAND IN A PLASTIC INDUSTRY}

\author{
Márcio Botelho da Fonseca Lima ${ }^{1}$; Luiz Bueno da Silva ${ }^{2}$; Ildo Fernandes Gomes ${ }^{3}$ \\ ${ }^{1}$ Universidade Federal da Paraíba-UFPB -João Pessoa - Brasil \\ tismalu@uol.com.br \\ ${ }^{2}$ Universidade Federal da Paraíba-UFPB -João Pessoa - Brasil \\ bueno@ct.ufpb.br \\ ${ }^{3}$ Universidade Federal da Paraíba-UFPB -João Pessoa - Brasil \\ producao@ct.ufpb.br
}

\begin{abstract}
Resumo
Este artigo tem como objetivo apresentar duas metodologias prospectivas e suas principais aplicações. Primeiro, apresenta-se o Método DELPHI, utilizado especialmente na elaboração de políticas de inovação e em ações de gestão nos níveis macro, de governo, meso, de Instituições de Ensino e Pesquisa, e micro, de empresas, num horizonte de planejamento a longo termo. Segundo, após uma breve explanação sobre séries temporais, desenvolve-se um modelo auto-regressivo para previsão de demanda de uma indústria de plástico da Paraíba. Concluindo, avaliou-se que o modelo auto-regressivo de segunda ordem era o mais ajustado à série de dados da empresa e verificou-se ainda que a previsão de vendas, embora oscilando fortemente ao longo do tempo, estimou valores próximos à demanda real, indicando a utilidade da Metodologia de Box-Jenkins para previsões de curto prazo. Para um horizonte de planejamento em longo prazo, recomenda-se a utilização do método DELPHI.
\end{abstract}

Palavras-chave: método Delphi, política de inovação, metodologia de Box-Jenkins.

\section{Introdução}

Na Grécia Antiga, especialmente na ilha de Delphos, sacerdotisas tornaram-se conhecidas por suas habilidades em prever o futuro. Uma das metodologias de prospectiva foi denominada DELPHI, em alusão a esta passagem histórica, e será apresentada inicialmente neste trabalho.

Em seguida, ainda no domínio das metodologias de prospectiva, desenvolve-se um modelo auto-regressivo para previsão de demanda, em uma indústria de plástico no Estado da Paraíba. 
Este modelo procura reduzir as incertezas (fazer previsões) decorrentes de situações complexas, antecipando e tentando vislumbrar possibilidades de processos, mediante o tratamento adequado de informações disponíveis.

Isso requer a utilização de uma abordagem sistêmica, com o propósito de simular os impactos produzidos por mudanças simultâneas de um conjunto de variáveis (ROCHA NETO, 2003).

Uma tendência tradicional de quem observa a evolução dos processos sociais tem sido aquela de imaginar que as mudanças ocorrerão da mesma forma como ocorreram no passado. Desta maneira, tende-se a conjeturar conseqüências a partir do presente para determinar o que acontecerá no futuro. Geralmente, esse enfoque determinista conduz a previsões equivocadas, especialmente devido a não linearidade dos processos de inovação.

Saliente-se que as atividades prospectivas apenas melhoram a qualidade dos processos decisórios, ampliando ou reduzindo o leque das escolhas possíveis, mas não devem restringir a liberdade de opção dos gestores.

Conceitualmente falando, Bergson (1859-1941) ressalta que tempo é processo, ou seja, é justamente aquilo que impede que tudo seja dado de uma só vez. Prognósticos são realizados por intermédio de um tempo representacional, inerente à lógica da retrospecção, que transforma a idéia de algo dado no presente como aquilo que no passado já existia, sob a forma de possibilidade. Assim, se a realidade presente já estava contida no passado, também a realidade futura deverá estar contida no presente, estando ambas as afirmações sob o mesmo signo da possibilidade. Mas se tempo é processo, a existência do tempo não provaria que há indeterminação nas coisas?

$\mathrm{O}$ fato de eliminar a duração do tempo corresponde a concebê-lo em termos representacionais, como sucessão de instantâneos justapostos. Isto é, algo fixo, portanto imóvel e passível de mensuração (JOHANSON, 2008).

Mas se a Mecânica, a Física e mesmo a Economia lidam com o tempo desse modo inerte, isso não é sem razão. Porque é preciso imobilizar, fixar o real para que dele se possam fazer previsões e intervenções nos processos de tomada de decisão.

No entanto, essa perspectiva de ação não é a mesma da perspectiva do conhecimento, ou seja, que se por um lado é justificável conceber o tempo em termos espaciais, em virtude das necessidades práticas, por outro lado isso não significa que essa seja sua realidade.

Em suma, o desenvolvimento progressivo do pensamento aberto pelos gregos acaba por delimitar um lugar inferior na teoria do conhecimento moderna para a experiência consciente, caracterizada por pretender recuperar o valor dos dados imediatos da experiência sensível A substituição desta experiência por um extrato fixo, vazio e consistindo em um sistema de idéias 
gerais abstratas identifica-se ao ato de alijar a sensibilidade no campo da teoria do conhecimento (MORATO PINTO, 2008).

\section{O Método DELPHI e Suas variações}

Em matéria de instrumentos de previsão qualitativa, o método "Delphi" é reconhecido como sendo um dos melhores. Sua área de atuação mais freqüente é a previsão tecnológica ou política de inovação, mas paulatinamente seu uso vem se estendendo para outros domínios como a Administração, especialmente no que tange à Descrição de Cenários Futuros no campo do Planejamento Estratégico. Sua utilização é mais indicada quando não se dispõem de séries de dados históricos relativos ao problema que se pretende investigar, ou seja, quando faltam dados quantitativos que lhe são referentes, ou quando há rupturas sociais, tecnológicas, políticas ou econômicas, como parece acontecer com grande intensidade neste quarto trimestre de 2008. Além dessas mudanças, constata-se a relevância do uso da Internet para a realização de discussões em grupo, tornando-se dessa maneira uma importante aliada na aplicação da Metodologia Delphi.

O fundamento do método se caracteriza por ser intuitivo e interativo. Implicando a constituição de um grupo de especialistas em determinada área do conhecimento, os quais respondem a uma série de questões cujos resultados são analisados, calculando-se a média, a mediana e a média das juntas, isto é, a média entre o primeiro e o terceiro quartil em um conjunto de dados. O resumo dos resultados é comunicado aos componentes do grupo que respondem novamente ao questionário. As interações se sucedem de tal maneira até que um consenso ou quase consenso seja obtido. As interações do método são denominadas rodadas ou rounds.

Assume-se que grupos de especialistas agregam uma quantidade mais elevada de conteúdos de informação relevantes que qualquer um de seus membros tomados individualmente.

Além disso, grupos cujos membros permanecem anônimos tendem a contribuir de maneira mais independente e menos vulnerável às pressões de ordem psicológica e cultural do que quando um grupo de especialistas é postos face-a-face para discutir um tema qualquer.

A técnica de capacitação pela seqüência DELPHI se caracteriza pelos seguintes princípios: anonimidade; interação com realimentação monitorada; obtenção de respostas estatisticamente processadas; e identificação de consensos e contraditórios.

Saliente-se que, durante a implantação da técnica DELPHI, os membros de um grupo não são informados a respeito da identificação dos demais participantes. De preferência, a interação deve ser conduzida de forma anônima, mediante a utilização de questionários respondidos a distância, via terminal de computador (MASSAUD, 2008). 
Observa-se que a maior parte dos processos de planejamento estratégico privilegia a obtenção de consenso, relegando a riqueza dos contraditórios, que talvez possam responder de maneira superior às questões mais relevantes. Os resultados são quantitativamente processados, obtendo-se afinal as opiniões da maioria, sem, no entanto, desconsiderar o registro dos contraditórios. O método Delphi deve ser subdividido em dois tipos de estudo: uma pesquisa exploratória e outra prospectiva.

A amostra utilizada em uma pesquisa exploratória poderá ser probabilística, intencional ou uma combinação de ambas. A amostra da pesquisa prospectiva também, sendo constituída pelos participantes da amostra do estudo exploratório que devolveram o questionário composto de questões fechadas, devidamente preenchidas. As rodadas do estudo prospectivo basear-se-ão em questionários compostos por questões abertas.

Nas linhas que se seguem, um roteiro para realização de uma seqüência DELPHI é apresentado - cada experimento é relacionado com uma rodada.

Além de perguntas, os questionários devem transmitir informações sobre o nível de consenso ou de contradição, bem como precisam servir para socializar os argumentos apresentados.

A primeira rodada deve ser aberta e não muito estruturada (brainstorming/brainwriting) de modo a induzir os participantes a apresentarem seus temores e suas idéias sem limitações.

Após coletar as contribuições obtidas na primeira rodada, o coordenador deve resumi-las e consolidá-las na forma de uma lista de questões consideradas prioritárias sobre o assunto. Em seguida, deve-se realizar um processo de organização dos resultados, mas ainda sem obter a avaliação crítica dos produtos obtidos.

Na segunda rodada, os participantes devem tecer as considerações que os levaram a escolher as questões listadas. Depois de coletar as contribuições dessa segunda rodada, o coordenador deve efetuar um resumo estatístico que será devolvido aos participantes na terceira rodada.

$\mathrm{Na}$ terceira rodada, os participantes são induzidos a rever ou confirmar suas posições anteriores, acrescentando argumentações e dados adicionais. O coordenador também deverá elaborar novo resumo estatístico, que deverá ser desenvolvido aos participantes juntamente com a síntese dos argumentos em conflito.

Entre as diferentes rodadas explicitadas acima, são realizados processos de critica e avaliação. É imprescindível que este método tenha, num primeiro momento, características de um laboratório. Servindo, desta maneira, como modelagem de instrumentação. Nesse caso, vale ressaltar, deve-se contar com a colaboração de colegas de trabalho do Departamento de Engenharia de Produção, por exemplo.

Segundo as recomendações de Dagnino (2002), relativas ao estabelecimento de políticas de inovação, geralmente, os projetos devem ter como objetivos gerais: 
- Ampliar a capacidade de formulação e implantação de políticas voltadas ao desenvolvimento científico e tecnológico no nível macro (governo do Brasil e dos Estados), no nível meso (Universidades e Fundações de Apoio à Pesquisa dos Estados) e micro (das empresas);

- Reforçar o potencial de pesquisa e formação de recursos humanos das Universidades, por intermédio da identificação e promoção de oportunidades de inovação;

- Aumentar o impacto desse potencial no contexto sócio-econômico externo a essa Instituição, mediante cooperação com outras organizações públicas e privadas.

A título de exemplo, a seguir, será explicitado um projeto em andamento na Universidade Federal da Paraíba (UFPB), denominado "Gestão da Inovação nos laboratórios do Centro de tecnologia (CT) da UFPB", com os seguintes objetivos:

Objetivo Geral - Avaliar e estimar quando serão implantadas certas diretrizes da Lei de Inovação de dezembro de 2004.

Para os efeitos dessa Lei, considera-se como inovação a introdução de novidade ou aperfeiçoamento no ambiente produtivo ou social que resulte em novos produtos, processos ou serviços. Além disso, entende-se como criação: invenção, modelo de utilidade, desenho industrial, programa de computador, topografia de circuito integrado e qualquer outro desenvolvimento tecnológico que acarrete ou possa acarretar o surgimento de novo produto, processo ou aperfeiçoamento incremental, obtido por um ou mais criadores. Enfim, o Núcleo de Inovação Tecnológica (NIT) é apreendido como núcleo ou órgão que tem como finalidade gerir a política de inovação da Instituição Científica e Tecnológica (ICT), órgão da administração pública que tenha como missão institucional executar pesquisa básica ou aplicada de caráter científico ou tecnológico.

\section{Objetivos Específicos}

- Verificar se os laboratórios do CT compartilham equipamentos, instrumentos e demais instalações com micro e empresas de pequeno porte, visando à incubação de empresas, bem como a utilização dessa infra-estrutura de pesquisa com empresas nacionais e organizações de direito privado sem fins lucrativos voltadas a atividades de pesquisa.

- Opinar sobre a conveniência de promover a proteção das criações desenvolvidas nos laboratórios do CT.

- Opinar sobre a conveniência de divulgação das criações desenvolvidas no CT, passíveis de proteção intelectual.

- Verificar se o NIT acompanha o processamento dos pedidos e a manutenção dos títulos de propriedade intelectual dos pesquisadores dos laboratórios do CT.

\subsection{Estado da Arte}


Sob o ponto de vista teórico, uma pesquisa bibliográfica preliminar foi empreendida, notadamente sobre a relação universidade - empresa. Por exemplo, a necessidade de se realizar uma abordagem comparativa das contribuições de Dagnino (2003), Dagnino e Dias (2007), Haase et al (2005) e Ruiz (2005), entre outros.

Com efeito, a título de ilustração, Dagnino (2003) aponta alguns indicadores que mostram a relação periférica do Brasil em comparação com os países avançados e os emergentes asiáticos: o dispêndio em P\&D da empresa privada nacional e o das transnacionais que operam no país (relativo ao PIB, ao dispêndio nacional em C\&T, ao faturamento) são notoriamente reduzidos; o desinteresse das empresas locais por atividades de P\&D; o desinteresse da empresa local pela baixa utilização pela empresa local das universidades e institutos de pesquisa públicos.

Por sua vez, Dagnino e Dias (2007) afirmam que o crescimento quantitativo da pesquisa científica não gera desenvolvimento tecnológico, pois enquanto que a produção científica nacional de 1990 a 2000 cresceu de 3555 artigos para 9511, nesse mesmo período as patentes brasileiras depositadas nos EUA passaram de 41 para 119. Assim, o Brasil produz um volume de conhecimento científico assimétrico em relação à tecnologia produzida, medido pelo número de patentes depositadas.

Eles argumentam também que doutores produzem artigos científicos; a produção de patentes é responsabilidade da empresa privada. Com efeito, o comportamento da produção de patentes não está ligado à produção de artigos científicos, mas sim ao investimento privado em P\&D. Por exemplo, a Coréia do Sul - por intermédio de investimentos consideráveis por parte de suas empresas, sobretudo a partir do final dos anos 80 - depositou 225 patentes nos EUA em 1990, passando a 3314 em 2000. A partir daí, a participação desse país no total de patentes no mundo cresceu de $0,33 \%$ para $2,76 \%$. No mesmo período, para o caso brasileiro, a participação do país no total mundial de patentes passou de $0,06 \%$ para $0,1 \%$, valor ainda muito baixo, a despeito de, segundo a Capes, a produção científica nacional ter crescido $133 \%$ nos últimos 10 anos, atingindo em 2007 a cifra de 26369 artigos em publicações estrangeiras, representando 1,75\% da produção mundial.

Ao contrário, Haase et al. (2005) apresentam determinadas influências das patentes sobre as universidades e o setor privado e as seguintes sugestões de políticas: melhoria da infra-estrutura de informações sobre patentes e sua difusão para o mercado; maior participação dos pesquisadores nas estratégias de exploração comercial; direcionamento das atividades de pesquisa à demanda do mercado; cooperação de firmas em atividades de pesquisas intensivas em P\&D; concessão de licenças exclusivas às empresas e otimização da concessão de patentes por intermédio de sistemas flexíveis de estímulos e redes de contacto. 
No mesmo sentido, Ruiz (2005), utilizando dados da European Patent Office entre 1978 e 2002, procura avaliar em que medida as universidades européias estão efetuando mudanças em sua missão pública, seja pela influência da regulamentação americana, seja pela aparição de novos elementos que modificaram o Sistema Nacional de Inovação, tais como o surgimento de novas ciências aplicadas (microbiologia), e a necessidade de se obter financiamento para $o$ desenvolvimento da pesquisa universitária e o fortalecimento das relações universidade-empresa.

Enfim, concluindo esta seção, apesar de se constituir num método muito poderoso de prospectiva tecnológica que será utilizado no projeto supracitado, o método DELPHI mostra um elevado grau de subjetividade das instruções obtidas, além de um exagerado otimismo da maioria dos especialistas participantes. Para atenuar essas deficiências, sugere-se a adoção suplementar de métodos quantitativos no que tange à análise de séries temporais, no âmbito da área de Engenharia de Produção, especialmente em Gerência de Projetos, na qual a técnica DELPHI é também utilizada para planejamento de riscos (HELDMAN, 2006).

\section{Técnica quantitativa - a metodologia de séries temporais}

Uma das técnicas quantitativas mais difundidas é a metodologia de séries temporais. Os modelos de séries temporais partem da idéia de que cada valor da série temporal pode ser explicado por valores prévios, a partir do uso da estrutura de correlação temporal que geralmente há entre os valores da série. Segundo Abdel-Aal e Al-Garni (1997), os modelos de séries temporais têm sido largamente utilizados para modelagem e previsão em aplicações médicas, ambientais, financeiras e de engenharia.

$\mathrm{Na}$ busca de considerar o comportamento futuro da demanda, algumas alternativas vinham sendo propostas pela empresa desse estudo de caso. Uma dessas alternativas é a aplicação de um modelo auto-regressivo aos dados históricos dessa empresa, com o objetivo de fazer a previsão da demanda de forma global de toda a família de produtos. Este modelo possibilitará subsidiar o planejamento da produção e alocação dos recursos, como mão-de-obra, mas de acordo com as variações no decorrer do ano, minimizando o impacto dessas variações na produtividade dessa empresa.

\section{Estudo de caso}

Localizada em João Pessoa, PB, e atuando no mercado brasileiro desde 1996, a RAVA Embalagens IND.COM. LTDA é uma empresa especializada na fabricação de embalagens, tanto para resíduos hospitalares, quanto comuns, com o principal objetivo de proporcionar a segurança ao processo de gerenciamento de resíduos sólidos, visando à preservação da saúde e do meio ambiente. 
A previsão de vendas dessas embalagens entre Janeiro de 2002 e Dezembro de 2006 está apresentada na Figura 1 - período que será utilizado para a formulação do modelo proposto.

Figura 1 - Histórico de vendas de 2002 a 2006.

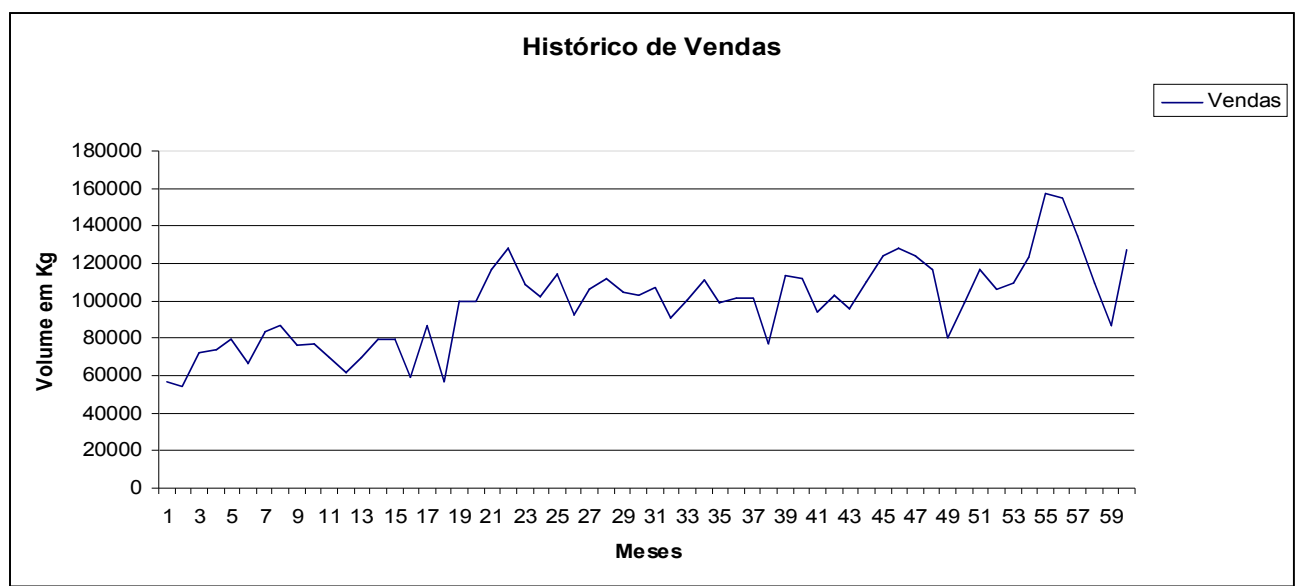

\subsection{Etapas da metodologia Box-Jenkins}

Segundo Morretin e Toloi (1987), a construção dos modelos Box-Jenkins é baseada em um ciclo iterativo, no qual a escolha do modelo é feita com base nos próprios dados. Segundo Box e Jenkins (1976), são três as etapas para construção do modelo: 1) Identificação: consiste em descobrir qual dentre as várias versões dos modelos de Box-Jenkins, sejam eles sazonais ou não, descreve o comportamento da série.

A identificação do modelo a ser estimado ocorre pelo comportamento das funções de autocorrelações (FAC) e das funções de auto-correlações parciais (FACP). Outros detalhes referentes à obtenção dessas funções podem ser pesquisados em Makridakis et al (1998); 2) Estimação: consiste em estimar os parâmetros $\phi$ e $\Phi$ do componente auto-regressivo, $\theta$ e $\Theta$ do componente de médias móveis e a variância de $\varepsilon t$; e 3) Verificação: consiste em avaliar se o modelo estimado é adequado para descrever o comportamento dos dados. Caso o modelo não seja adequado, o ciclo é repetido, voltando-se à fase de identificação. Um procedimento muito utilizado é identificar não só um único modelo, mas alguns modelos que serão então estimados e verificados. Quando se obtém um modelo satisfatório, passa-se para a última etapa da metodologia de Box-Jenkins, que constitui o objetivo principal da metodologia: realizar previsões.

\subsubsection{Etapa de identificação}

Para uma análise de dados de previsão de demanda, a partir de séries temporais, deve-se considerar a capacidade constante de venda durante o período apresentado na figura 1, pois uma 
alteração nesta capacidade pode invalidar a previsão. Assim, os dados foram considerados a partir do mês 19, formando então uma série de 42 observações, isto é, vendas entre julho de 2003 e dezembro de 2006 - período constante. Toda a análise de dados e a formulação do modelo foram realizadas no Laboratório de Métodos Quantitativos Aplicados do Departamento de Engenharia de Produção da UFPB.

Analisando o volume de vendas entre o mês de julho (1) de 2003 a dezembro (42) de 2006, observa-se uma tendência pouco crescente no volume de vendas (figura 2). Portanto, como a série apresenta inclinação muito pequena, ela será considerada estacionária.

Figura 2 - Vendas de julho de 2003 a dezembro de 2006 e sua tendência.

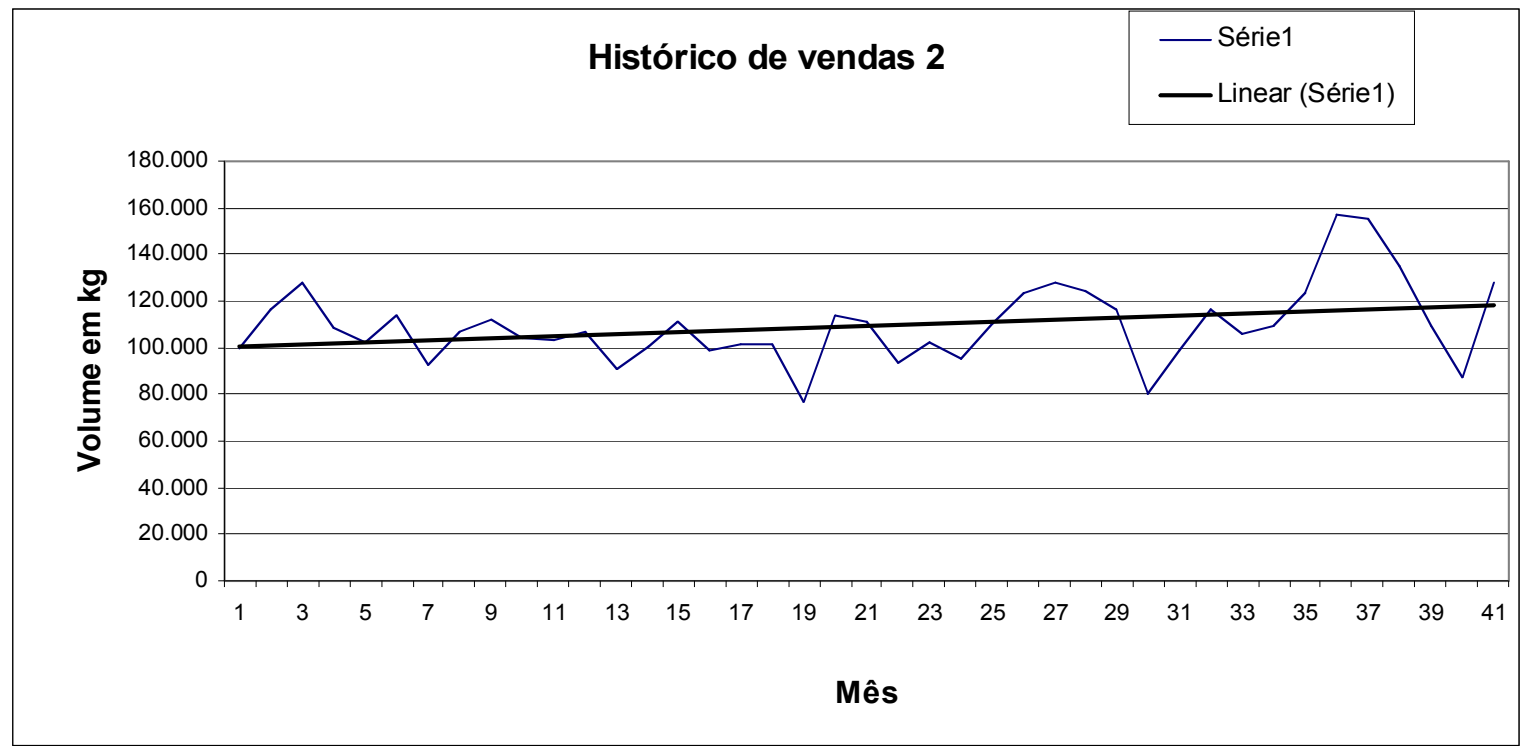

A figura 3 apresenta a FAC para o volume de vendas. Pode-se observar que esta decai exponencialmente rápida, chegando à zero nas primeiras defasagens, o que indica se tratar de um modelo ARIMA, isto é, AR(1). A FACP apresenta as auto-correlações parciais, as quais medem a correlação entre o valor de $\mathrm{Zt}$ e $\mathrm{Zt}-\mathrm{k}$, descontadas a influência dos valores de $\mathrm{Zt}-1$ até $\mathrm{Zt}-\mathrm{k}+1$ (figura 4). Observa-se que apenas a auto-correlação de defasagem 1 é significativa, reforçando a indicação de um modelo $\mathrm{AR}(1)$. Tendo em vista que a série pode ser considerada estacionária, a ordem de integração é zero, isto é, $\mathrm{d}=0$. 


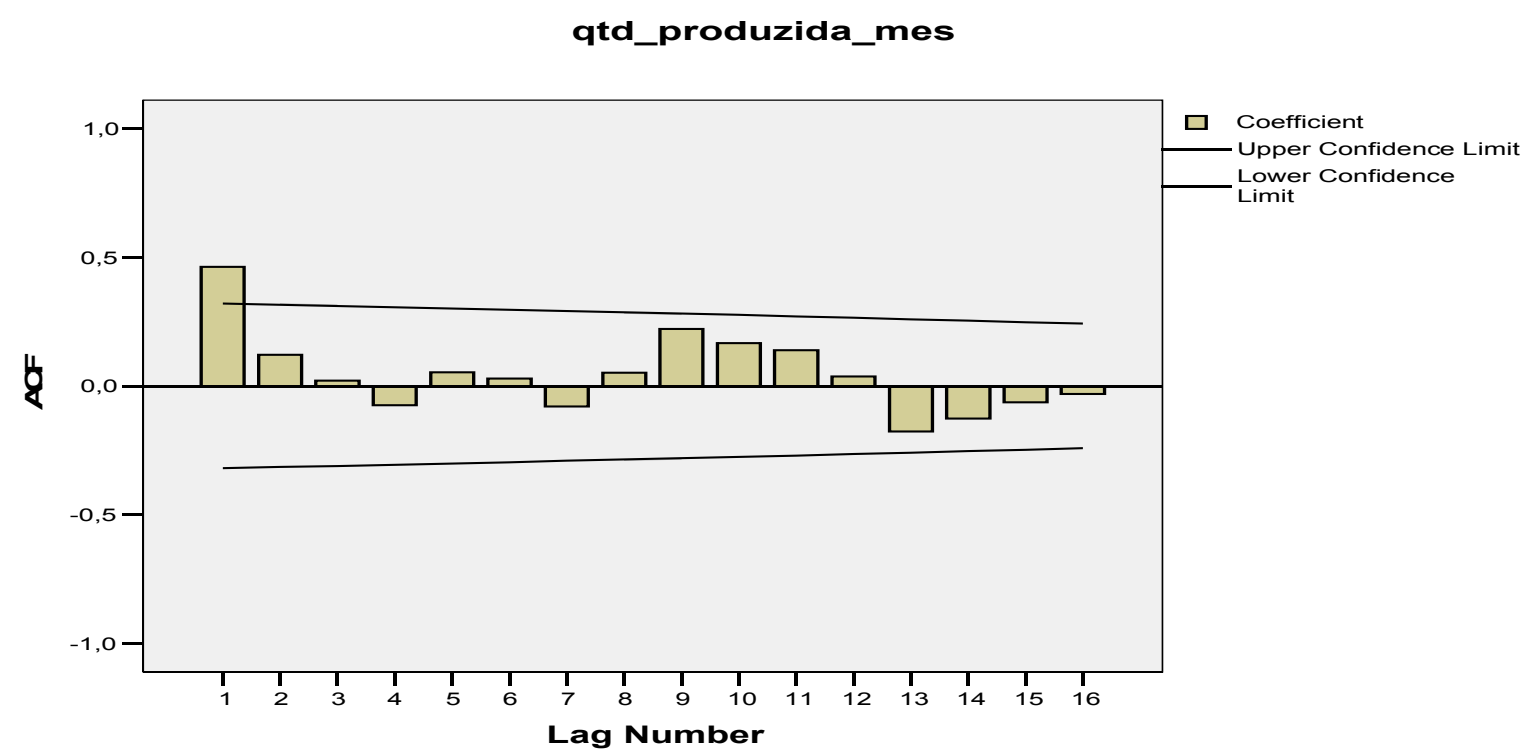

Figura 4 - Função Auto-Correlação Parcial (FACP) para o volume de vendas.

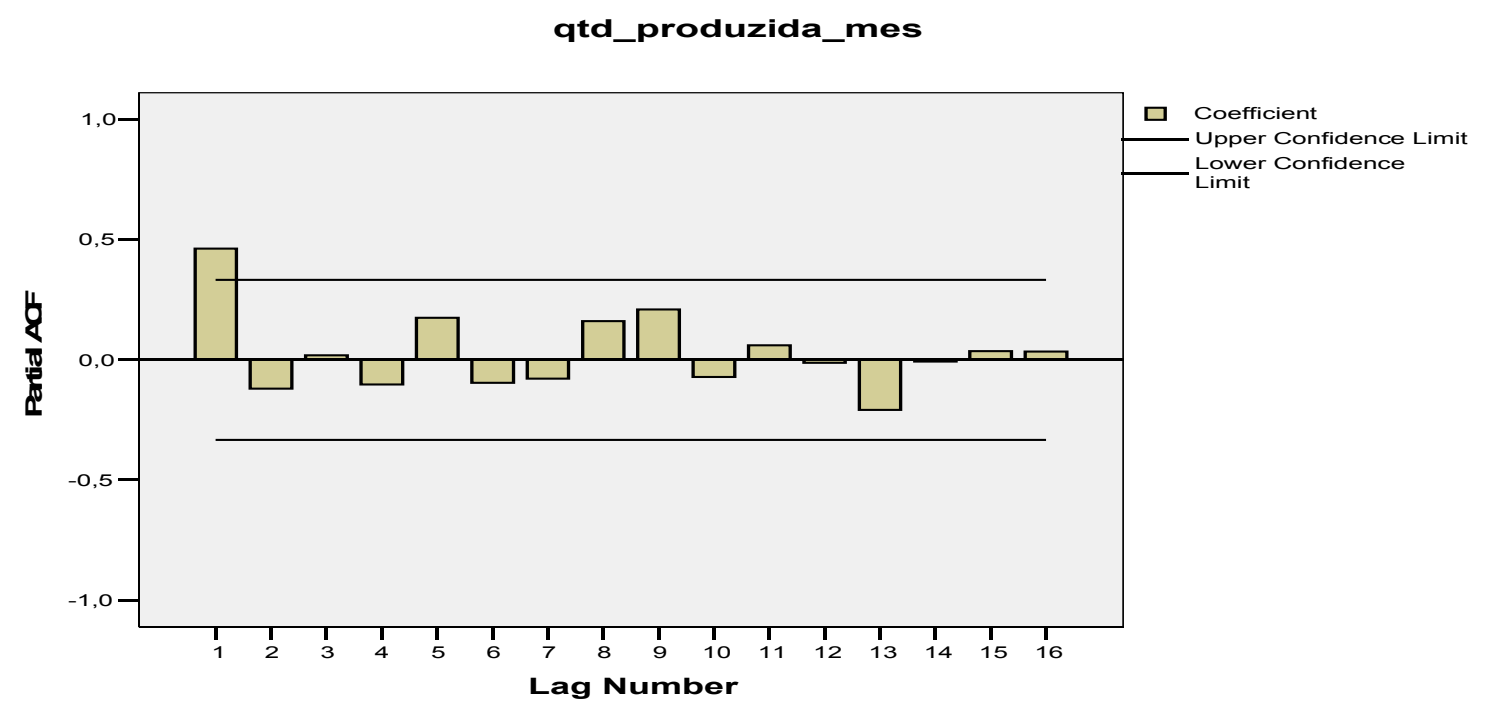

\subsubsection{Etapa de estimação}

Além dos parâmetros do modelo, cujos valores de $p=1$ (modelo auto-regressivo de primeira ordem), $d=0$ (a série foi considerada estacionária, não sendo necessária nenhuma diferenciação) e $q=0$ (não foi identificado nenhum parâmetro relativo às médias móveis), estimar-se-ão parâmetros de mais um modelo para posteriormente realizar uma análise comparativa, identificando qual realmente será o melhor modelo para fazer a previsão. As estimativas dos parâmetros dos modelos estão descritos na tabela 1 . 
Tabela 1 - Estimativa dos parâmetros dos modelos propostos para comparação

\begin{tabular}{|c|r|r|}
\hline Parâmetros estimados & AR (1) & AR (2) \\
\hline$\varnothing_{1}$ & 0,999 & 1.084 \\
\hline$\varnothing_{2}$ & & $-0,084$ \\
\hline$c$ & 20,967 & 20,966 \\
\hline
\end{tabular}

Fonte: Software SPSS (versão 13.0)

Assim, a equação (1) representa o modelo AR (1) e a equação (2) representa o modelo AR (2).

$$
\begin{aligned}
& \mathrm{Y}_{\mathrm{t}}=20,96+0,999 \mathrm{Y}_{\mathrm{t}-1} \\
& \mathrm{Y}_{\mathrm{t}}=20,96+1,084 \mathrm{Y}_{\mathrm{t}-1}-0,084 \mathrm{Y}_{\mathrm{t}-2}
\end{aligned}
$$

\subsubsection{Etapa de verificação}

Essa etapa consiste em verificar se o modelo identificado e estimado é adequado.

a) Avaliação por meio da análise de resíduos

Os resíduos do modelo estimado $\hat{\varepsilon}_{t}$ são estimativas do ruído branco, sendo assim, devem apresentar auto-correlações não muito significantes. Para testar essa suposição, verificou-se o comportamento da FAC para os resíduos estimados. A figura 5 revela que os resíduos apresentam comportamento aleatório, ou seja, não revelam padrão específico. Assim, a análise dos resíduos confirma a adequação do modelo.

Figura 5 - Função de Auto-Correlação para os resíduos

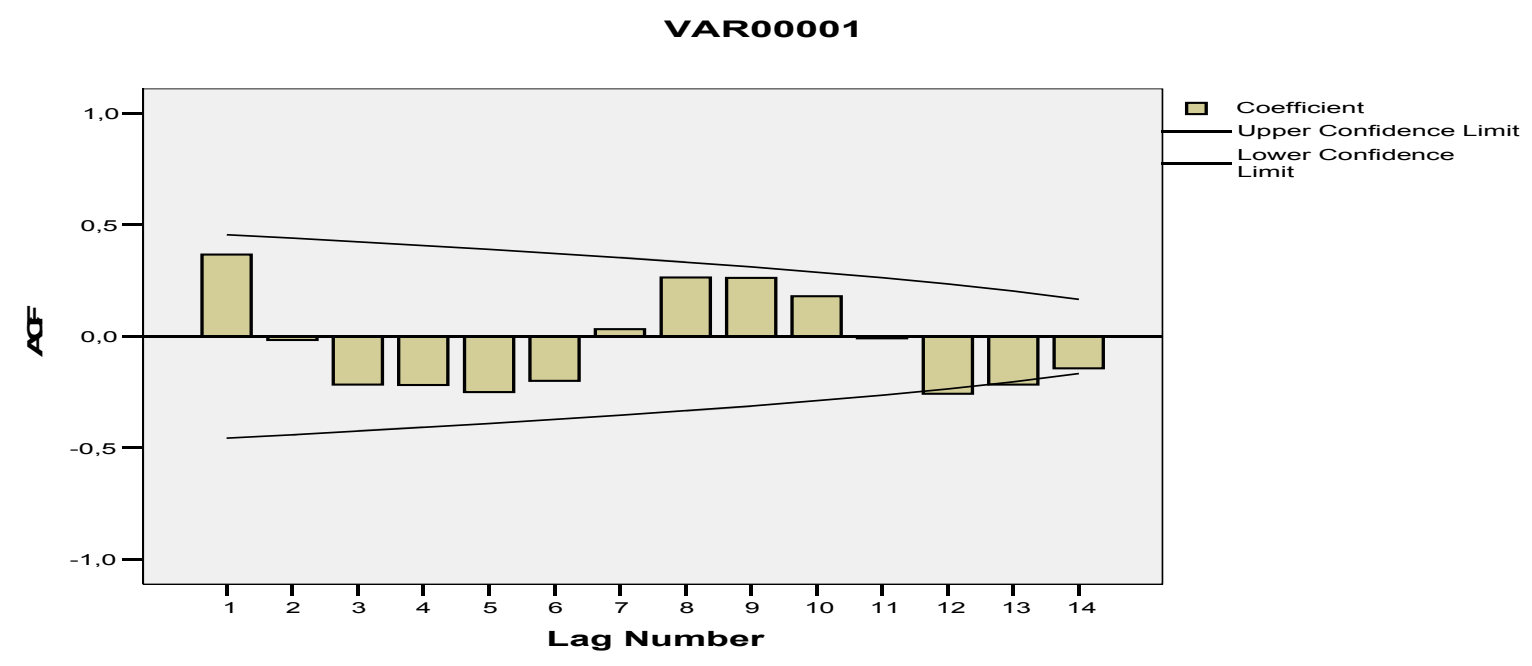

b) Avaliação da ordem do modelo

Para ratificar ou não o modelo AR (1) através da FAC, far-se-á uma análise comparativa da eficácia do mesmo em relação ao modelo AR (2). A tabela 2 apresenta uma comparação entre os modelos AR (1) e AR (2) proposto para o volume de vendas mensais. Para estes, os coeficientes (A) 
são significativos, ao nível de significância $\alpha=0,05$. A análise da variância residual é outro indicador para verificar se a ordem do modelo está adequada; quanto menor a variância, melhores serão as previsões. Os critérios de AIC (Akaike Information Criteria) e SBC (Schwartz Bayesian Criteria) também conhecido como BIC (Critério de Informação Byesiano), utilizados para comparação de modelos, leva em consideração a variância do erro; e, quanto menores forem os valores destes critérios, mais adequado será o modelo. Assim, observa-se na tabela 2 que os valores destes critérios e da variância residual do modelo AR (2) são menores que o do AR (1). Logo, o modelo AR (2) é o mais adequado para realizar as previsões de vendas mensais, o qual é representado pela equação 2 , isto é, $\mathrm{Y}_{\mathrm{t}}=20,96+1,084 \mathrm{Y}_{\mathrm{t}-1}-0,084 \mathrm{Y}_{\mathrm{t}-2}$.

Tabela 2 - Critérios de comparação entre os Modelos AR(1) e AR(2)

\begin{tabular}{|c|c|c|c|r|}
\hline Modelo & A & $\begin{array}{c}\text { Critério } \\
\text { AIC }\end{array}$ & $\begin{array}{c}\text { Critério } \\
\text { BIC }\end{array}$ & $\begin{array}{r}\text { Variância } \\
\text { residual }\end{array}$ \\
\hline AR(1) & $\varnothing_{1}=0,000$ & 128,334 & 133,474 & 1,038 \\
\hline AR(2) & $\begin{array}{l}\varnothing_{1}=0,000 \\
\emptyset_{2}=0,007\end{array}$ & 123,911 & 130,766 & 0,958 \\
\hline
\end{tabular}

Fonte: Software SPSS (versão 13.0)

\subsection{Etapa da previsão}

A tabela 3 apresenta dados observados e previstos para previsões dos meses de Janeiro-Julho de 2007.

Tabela 3 - Comparação entre dados observados e a previsão de vendas

\begin{tabular}{|c|c|c|c|}
\hline Mês & Volume vendido & $\begin{array}{c}\text { Volume estimado } \\
\text { Modelo AR(2) }\end{array}$ & erro \\
\hline 41 & 112386 & - & - \\
\hline 42 & 127603 & - & - \\
\hline 43 & 125698 & 128902,2 & 3204,2 \\
\hline 44 & 120489 & 125558,9 & 5069,9 \\
\hline 45 & 132846 & 120072,4 & 12773,6 \\
\hline 46 & 128643 & 133904,9 & 5261,9 \\
\hline 47 & 119654 & 128310,9 & 8656,9 \\
\hline 48 & 130954 & 118919,9 & 12034,1 \\
\hline 49 & 138964 & 131924,2 & $-7039,8$ \\
\hline
\end{tabular}


Nesta tabela nota-se que a média do erro ou a diferença média entre os valores observados e estimados pelo modelo AR (2) está em torno de 7.719,7 kg. Essa diferença representa apenas 6,1\% do total de vendas mensais, o que torna este modelo adequado para fazer tal previsão.

\title{
5. Considerações finais
}

Realizar previsão de demanda é uma atividade importante, pois pode revelar as tendências de mercado e contribuir para o planejamento estratégico da empresa. Tais previsões também auxiliam na solução de problemas mais imediatos, como a definição do volume de vendas dos próximos meses para atender a demanda com rapidez e qualidade, sem afetar o processo da empresa. Visando alcançar esses objetivos, este artigo apresentou algumas metodologias prospectivas, entre elas a metodologia de Box-Jenkins, aplicando-a por meio de um estudo de caso dirigido ao setor de vendas de uma indústria de embalagens plásticas.

Verificou-se que a demanda da empresa é muito variável, tornando difícil fazer uma análise mais simples para prever as vendas futuras. Assim, optou-se por essa metodologia, que poderá contribuir para um melhor planejamento da produção dessa empresa em curto prazo, com impacto na velocidade do atendimento a clientes e no equilíbrio de seu estoque. Mas sua aplicação requer atenção na escolha do modelo que melhor defina o comportamento temporal dos dados. Neste artigo, o modelo AR (2) foi o mais ajustado devido a sua menor variância residual e aos melhores critérios AIC e SBC. Este modelo auto-regressivo pode também ser utilizado para previsão de curto prazo da produção científica a ser gerada nos laboratórios e nos cursos de pós-graduação de uma Universidade Federal, por exemplo.

\begin{abstract}
The objective of this paper is to present two methodologies of forecast and its main applications. First, the DELPHI Method, used especially in the elaboration of innovation policies and in action of management in the levels macro, of government, meso, of Education and Research institutions, and micro, of companies, in a planning horizon of long term. Second, after one soon communication on time series, it is developed a auto-regressive model for forecast of demand of a plastic industry of the Paraíba. Concluding, it was evaluated that the auto-regressive model of second order was the most adjusted to the series of data of the company and was verified too that forecast of sales, even so oscillating strong throughout the time, estimated next values to the real demand, indicating the utility of the Methodology of Box-Jenkins for forecasts of short term. For a horizon of planning in long term, it is recommended the utilization of the Delphi method.
\end{abstract}

Keywords: Delphi method, policies of innovation, methodology of Box-Jenkins.

\section{Referências}

BOX, George E. P.; JENKINS, Gwilym M.; REINSEL, Gregory C. Time Series Analysis: forecasting and control. $3^{\mathrm{a}}$ ed. Englewood Cliffs: Prentice Hall, 1994. 
DAGNINO, Renato. A Relação Universidade-Empresa no Brasil e o Argumento da Hélice Tripla. Revista Brasileira de Inovação, v.4, nº. 2, julho/dezembro 2005.

DAGNINO, Renato. Gestão Estratégica da inovação: metodologias para análise e implementação. Taubaté, SP: Cabral Editora e Livraria Universitária, 2002.

DAGNINO, Renato; DIAS, Rafael. A Política de C\&T Brasileira: três alternativas de explicação e orientação. Revista brasileira de Inovação, v.6, $n^{\circ}$ 2, julho/dezembro 2007.

GONÇALVES, Reinaldo. A empresa Transnacional. In: Economia Industrial: Fundamentos Teóricos e Práticas no Brasil. Organizadores: David KUPFER e Lia HASENCLEVER. Rio de Janeiro:Elsevier, 2002.

HAASE, Heiko; de ARAUJO, Eliane C.; Dias Joilson. Inovações Vistas pelas Patentes: Exigências frente às novas funções das Universidades. Revista Brasileira de Inovação, v.4, nº. 2, julho/dezembro 2005.

HELDMAN, Kim. Gerência de projetos: PMP Project Management Professional. Rio de Janeiro: Elsevier, 2006.

JOHANSON, Izilda. Cem anos de Evolução. Revista Brasileira de Cultura, ano 11, no. 122, março 2008.

MAKRIDAKIS, Spyros; WHEELWRIGHT, Steven C; HYNDMAN, Rob J. Forecasting: methods and applications. $3^{\mathrm{a}}$ ed. New York: Wiley, 1998.

MASSAUD, Clovis; Metodologia Delphi. http://www.clovis.massaud.nom.br/prospec.htm.

MORATO Pinto, Débora C. A filosofia de Experiência na aurora do século 20. Revista Brasileira de Cultura, ano 11 , no. 123 , abril 2008.

MORETIN, Pedro A.; TOLOI, Clélia M. Séries Temporais. 2ª ed. São Paulo: Atual, 1987.

ROCHA NETO, Ivan. Gestão Estratégia de conhecimentos \& Competências: administrando incertezas e inovações. Brasília: ABIPTI/UCB/Universa, 2003.

RUIZ, Ana Urraca. Patentes y función Publica Universitária em Europa: Mitos y Realidades. Revista Brasileira de Inovação, v.4, n' 2 , julho/dezembro 2005.

Nome Completo: MARCIO BOTELHO DA FONSECA LIMA

Filiação institucional: Universidade Federal da Paraíba

Departamento: Departamento de Engenharia de Produção

Função ou cargo ocupado: Professor Assossiado II

Endereço: Rua Dr. Alcebíades Silva, 121/202, Cabo Branco, João Pessoa/PB. Cep: 58.045-340

Cep: 58.051-970

Telefone: (83) 32270381

e-mail: tismalu@uol.com.br

Nome Completo: LUIZ BUENO DA SILVA

Filiação institucional: Universidade Federal da Paraíba

Departamento: Departamento de Engenharia de Produção

Função ou cargo ocupado: Professor Adjunto IV

Endereço: Av. Acre, 130, apto 201, Ed. Rosa Leal, Bairro dos Estados, João Pessoa, PB.

Cep:58030-230 
Telefone: (83) 32444166

e-mail: bueno@ct.ufpb.br

Nome Completo: ILDO FERNANDES GOMES

Filiação institucional: Universidade Federal da Paraíba

Departamento: Departamento de Engenharia de Produção

Função ou cargo ocupado: Graduando em Engenharia de Produção

Endereço: Campus I-João Pessoa/PB-Cidade Universitária.

CEP: 58051-970

Telefone: (83)32167549

e-mail:producao@ct.ufpb.br

Recebido para publicação em: 26/02/2009

Aceito para publicação em: 13/03/2009 